\title{
Feasibility Study on Lifting of Seabed Materials Using a Bubble-Jet-Type Air-Lift Pump
}

\author{
Michio Sadatomi, Shingo Sugikubo, Daiki Goto, Kouhei Tajiri and Akimaro Kawahara \\ Department of Advanced Mechanical Systems, Graduate School of Science and Technology, Kumamoto University, Kumamoto \\ 860-8555, Japan
}

\begin{abstract}
The present study is concerned with the lifting of seabed materials by a BJT (bubble-jet-type) air-lift pump patented by Sadatomi. The targets are methane-hydrate rich muds on the bed about $200 \mathrm{~m}$ in depth around Japan islands and rare-earth rich muds on the bed deeper than 4,000 m around Minami-Torishima islands in the Pacific Ocean. Feasibility studies were conducted using $50 \mathrm{~mm}$ I. D. (inner diameter) and $5.0 \mathrm{~m}$ long vertical pipe as the pump upriser, VC (vinyl chloride) particles and natural sands mixture in the methane-hydrate case, and ceramics particles with $3,761 \mathrm{~kg} / \mathrm{m}^{3}$ in density in the rare-earth case as the deposits. From the methane-hydrate simulation experiments, an efficient operation condition with high VC particles to sands lifting ratio has been clarified. In the rare-earth case, the air supplies from two different midways in the upriser pipe have been tested together with the bottom supply because the air supply from the upriser bottom is very hard in deep sea. The effects of the air supply position on the pump performance have been clarified by the experiments and the simulations with a revised model applicable to the midway air supply type.
\end{abstract}

Key words: Hydraulic lifting, seabed materials, bubble-jet, air-lift pump, experiment, simulation.

\section{Introduction}

Air-lift-pump has been paid attention since it is usable to lift up light minerals deposited on seabed [1-9]. However, if a common air-lift pump shown in the left of Fig. 1 were used, it cannot pump up heavier sediments besides floating sediments. Thus, Sadatomi [10] invented a BJT (bubble-jet-type) air-lift pump with bubble-jet generators inside the skirt below the upriser as shown in the right of Fig. 1. By striking air-water mixture against the seabed or the lakebed with the bubble-jet generators, the BJT pump can lift up heavier deposits on the bed together with water and air, though the common air-lift pumps [2-4] cannot lift up them. Sadatomi and his colleagues have studied its usage for lifting deposits on the beds in aquafarm in sea and in reservoirs in order to purify water near the seabed and to increase water capacity in the reservoirs [11-14]. In addition, they proposed a model to predict the pump performance and validated the model against

Corresponding author: Michio Sadatomi, professor, Dr. Eng., research fields: fluids engineering and multi-phase flow. their experimental data on discharge rates of particles and water [15].

The present study is concerned with the lifting of seabed materials in a deep sea by the BJT pump. The targets are two kinds of materials: methane-hydrate rich muds on the bed about $200 \mathrm{~m}$ in depth around Japan islands and rare-earth rich muds on the bed deeper than 4,000 m around Minami-Torishima islands in the Pacific Ocean [16]. Since the specific gravity of the methane-hydrate ones is around unity while that of the rare-earth one around three, different efforts are needed for their efficient lifting. In a methane-hydrate

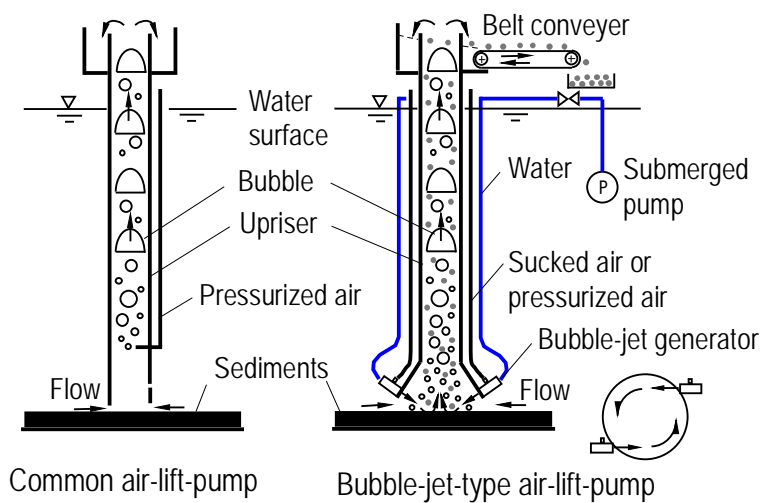

Fig. 1 Common and bubble-jet-type air-lift pumps. 
lifting simulation experiment, the methane hydrate rich mud was simulated by VC (vinyl chloride) particles, and a mixture of VC particles and sand was used as the test particles. The purpose of the experiment is to find an optimum operation condition, in which the lifting ratio of VC particles to sands is high. In a rare-earth lifting simulation experiment, the rare-earth mud was simulated by ceramics particles with $3,761 \mathrm{~kg} / \mathrm{m}^{3}$ in density. The purpose of the experiment is to clear the effects of submergence ratio and the air supply position in the air-lift upriser on the pump performance. In addition, another purpose of the rare-earth case is to clear the above effects by a numerical simulation by a model revised in the present study. Such results on two-kinds of experiments and on the simulations are described in the present paper.

\section{Methane Hydrate Lifting Simulation}

\subsection{Experiment}

Fig. 2 shows the present apparatus, the same as that used in our previous studies $[14,15]$. The upriser of the pump is a transparent acrylic pipe of $D=50 \mathrm{~mm}$ inner diameter and $H=5.0 \mathrm{~m}$ long. After setting the pump at a submergence ratio of $\sigma=H_{S} / H=0.76$, water was pumped from the bottom of the lower tank to the four BJ generators, while air was supplied from a compressor. The inlet volume flow rates of water and air to the BJ generators, $Q_{L I}$ and $Q_{G I}$, were measured with turbine flow meters and rotameters, within the accuracies of $1 \%$ and $2 \%$, respectively. The water jet with air bubble from the BJ generators attacked the particles bed, and a part of the particles beneath the skirt was floated and sucked into the upriser with water and air bubbles. They flowed up in the upriser and discharged into a separator, and air was released to atmosphere while the particles were separated from water with a net. The water flow rate was measured with an electromagnetic flow meter while the particles flow rate with a beaker and a stopwatch, both within the accuracy of $2 \%$. After the measurements, water and the particles were returned to the lower tank via a

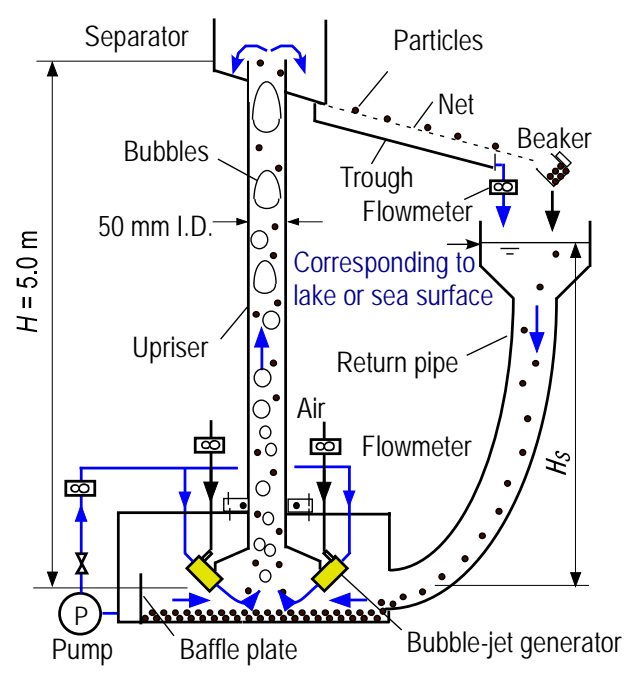

Fig. 2 Experimental apparatus (bottom type).

return pipe in order to allow steady lifting of the particles and the water.

Fig. 3 shows the BJ generator used in the present experiment. Water was supplied from the right, while air from the top through 24 holes of $1.0 \mathrm{~mm}$ diameter. The diameters of the pipe and the orifice were $D=11$ $\mathrm{mm}$ and $d_{O}=7.2 \mathrm{~mm}$. Four BJ generators were equipped inside the $415 \mathrm{~mm}$ O.D. skirt with the pitch diameter of $L_{p}=250 \mathrm{~mm}$ at the bottom of the upriser, which was confirmed to be the best pitch in our preliminarily test.

Table 1 lists specifications of two-kinds of particles in methane hydrate simulation test, and Fig. 4 is the picture of the VC particles and sand mixed one to one in volume in the lower tank. Their mean diameters and densities were different, but the free fall velocities

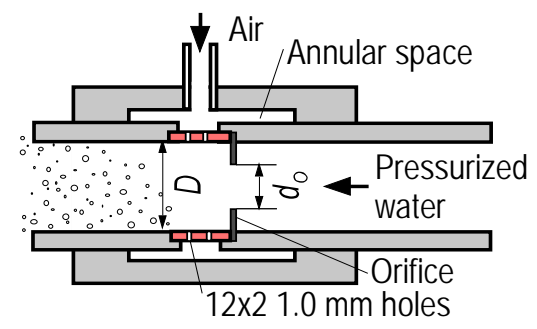

Fig. 3 Bubble-jet generator.

Table 1 Particles in methane hydrate lifting test.

\begin{tabular}{llllll}
\hline Name & $\begin{array}{l}\text { Mean dia. } \\
(\mathrm{mm})\end{array}$ & $\begin{array}{l}\text { Density } \\
\left(\mathrm{kg} / \mathrm{m}^{3}\right)\end{array}$ & $\begin{array}{l}\text { Free fall } \\
\text { vel. }(\mathrm{m} / \mathrm{s})\end{array}$ & $R e$ & $C_{D}$ \\
\hline Sand-1.6 & 1.55 & 2,600 & 0.18 & 284 & 0.96 \\
VC-3.9 & 3.92 & 1,377 & 0.17 & 666 & 0.54 \\
\hline
\end{tabular}




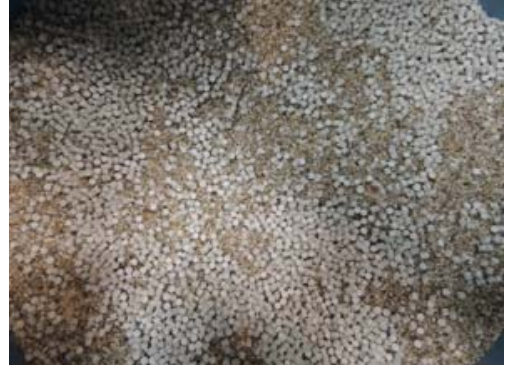

Fig. 4 VC and sand mixture as test particles.

measured in stagnant water were nearly the same. Also listed is the particle Reynolds number and the drag coefficient obtained from the free fall velocity and the particle mean diameter data.

The total power consumed by the BJT pump, $L_{T}$, was determined as the sum of the water power, $L_{L}$, and the pneumatic power, $L_{G}$. They were calculated from the following equations [15] by substituting the data on the water and air pressures and supply rates at $\mathrm{BJ}$ generator inlet, $P_{L I}, P_{G I}, Q_{L I}, Q_{G I}$, and their mean velocities, $u_{L I}$ and $u_{G I}$ :

$$
\begin{aligned}
& L_{L}=\left(P_{L I}+\rho_{L} u_{L I}^{2} / 2\right) Q_{L I} \\
& L_{G}=\left(P_{G I}+\rho_{G} u_{G I}^{2} / 2\right) Q_{G I}
\end{aligned}
$$

Here, $L_{L}$ and $L_{G}$ are the densities of water and air at the $\mathrm{BJ}$ generator inlet.

\subsection{Experimental Results}

As the first step, experiments were conducted by filling one of two kinds of particles in the lower tank of the BJ air-lift pump. Figs. 5a and 5b show a typical test result on the particles and water discharge rates, $Q_{S O}$ and $Q_{L O}$, against the air supply rate, $Q_{G I}\left(=Q_{G O}\right)$, at a fixed submergence ratio of $\sigma=0.76$. $Q_{S O}$ and $Q_{L O}$ data when VC-3.9 alone and sand-1.6 alone was filled are simultaneously plotted against air supply rate, $Q_{G I}$ on the respective figures. The data were taken at a fixed water supply rate of $Q_{L I}=52 \mathrm{l} / \mathrm{min}$ for VC-3.9 and 84 1/min for sand-1.6, because the highest $Q_{S O}$ values were obtained at the above $Q_{L I}$ conditions from a preliminary test. As $Q_{G I}$ increases, $Q_{S O}$ and $Q_{L O}$ increase because the mixture density of particles, water and air decreases and gives higher driving force to the air-lift pump. $Q_{S O}$ data for VC-3.9 case are nearly the same as those for sand-1.6 case, meaning a similar floating effect to VC-3.9 and sand-1.6 by the bubble jet. In addition, $Q_{L O}$ data are higher in VC-3.9 case than sand-1.6 case, because of lower particle density, leading lower mixture density of three-phases and higher driving force.

Fig. 6 shows $Q_{S O}$ data against total power supplied from air and water sources, $L_{T}$. If $L_{T}$ is lower than 0.23 $\mathrm{kW}, Q_{S O}$ is much higher for VC-3.9 than sand-1.6. This suggests us if $L_{T}$ were in a lower region than, say, 0.17 $\mathrm{kW}$ (correspond to $Q_{L I}=60 \mathrm{l} / \mathrm{min}$ ), lighter particle such as methane hydrate alone can be lifted.

As the second step, experiments were conducted by filling two kinds of particles together in the lower tank, and $Q_{L I}$ was reduced systematically as $60,50,40 \mathrm{l} / \mathrm{min}$ as a trial at $\sigma=0.76$. The experimental data on $Q_{S O}$ were obtained by changing the air supply rate at each $Q_{L I}$. As a result, the difference of $Q_{S O}$ between VC-3.9 and sand-1.6 was large at $Q_{L I}=40 \mathrm{l} / \mathrm{min}$ in Fig. 7, while it was small at $Q_{L I}=60$ and $50 \mathrm{l} / \mathrm{min}$.

Fig. 8 shows the discharge rate ratio of VC-3.9 to sand-1.6 at $Q_{L I}=40 \mathrm{l} / \mathrm{min}$ and $\sigma=0.76$. The ratio

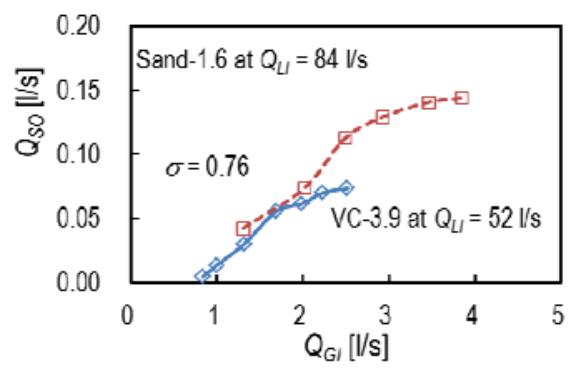

(a)

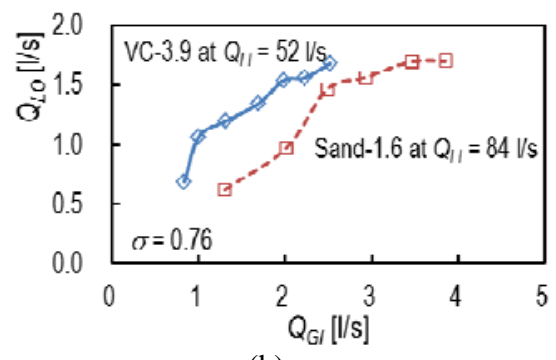

(b)

Fig. 5 Solid and water discharge rates against air supply rate from BJ generator, when VC-3.9 or sand-1.6 particles alone were fed in the lower tank. 


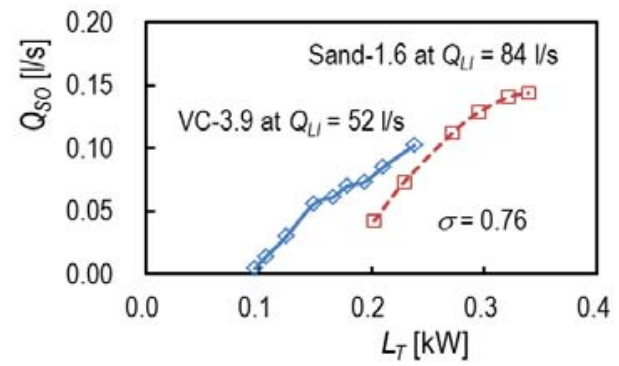

Fig. 6 Solid discharge rates against total power, when VC-3.9 or sand-1.6 particles alone were in the lower tank.

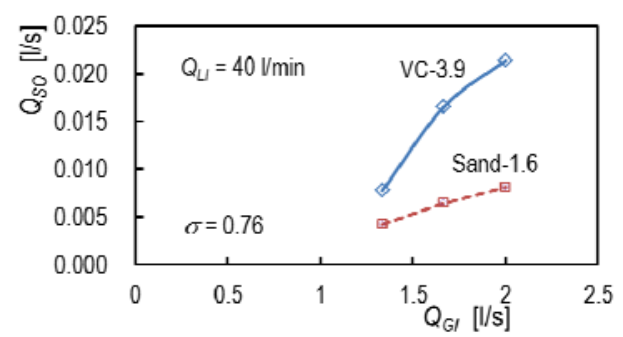

Fig. 7 Solid discharge rates for VC-3.9 particles and sand-1.6 particles against air supply rate, when both particles were fed in the lower tank.

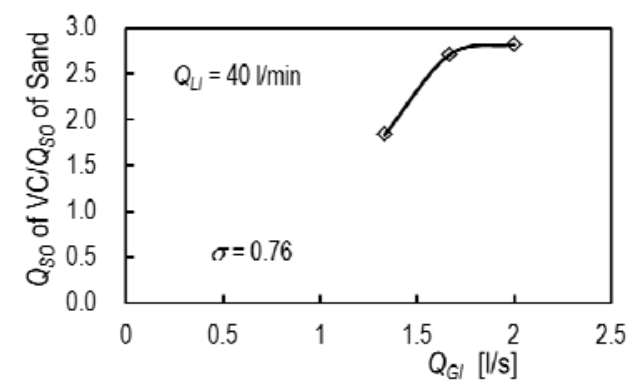

Fig. 8 Solid discharge rate ratio of VC-3.9 to sand-1.6 at $Q_{L I}=40 \mathrm{l} / \mathrm{min}$ and $\sigma=0.76$.

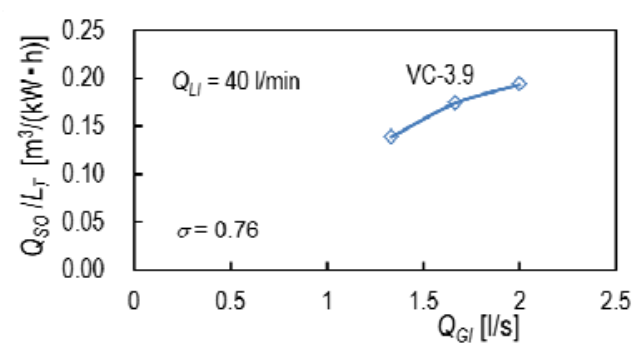

Fig. 9 Ratio of solid discharge rate of VC-3.9 to total power at $Q_{L I}=40 \mathrm{l} / \mathrm{min}$ and $\sigma=0.76$.

increased with the air supply rate and approached to 2.8 when $Q_{G I}>1.7 \mathrm{l} / \mathrm{s}$.

Fig. 9 shows the ratio of the discharge rate of VC-3.9 to the total power, $Q_{S O} / L_{T}$ against the air supply rate. The ratio is higher the better in view of energy saving. Thus, it can be concluded that the lifting at $Q_{G I}=2.0 \mathrm{l} / \mathrm{s}$ and $Q_{L I}=40 \mathrm{l} / \mathrm{min}$ is the best operation condition for VC-3.9 lifting at $\sigma=0.76$.

\section{Rare-Earth Lifting Simulation}

\subsection{Experiment}

Kato et al. [16] reported the existence of REY (rare-earth and yttrium) rich mud (0.2 wt\% in REY concentration) on seabed deeper than 4,000 $\mathrm{m}$ around Minami-Torishima islands in the Pacific Ocean. Since the mean density and the size of the mud have never been reported, we used a spherical ceramics particle, Cer-1, as the test particles for simulating the rare-earth lifting. As listed in Table 2, Cer-1 particles have 1.45 times higher density than common sand.

Since air supply to the deep seabed is not easy, we added a midway air supply type shown in Fig. 10 in addition to the bottom air supply type in Fig. 2. In the midway type, the distance from the air supply position to the bottom, $L_{E}$, was changed as $1.0 \mathrm{~m}$ and $1.4 \mathrm{~m}$, and water alone was jet from the BJ generators for particle floatation. So, a liquid-solid two-phase flow and a gas-liquid-solid three-phase flow coexisted below and

Table 2 Particles in rare-earth lifting test.

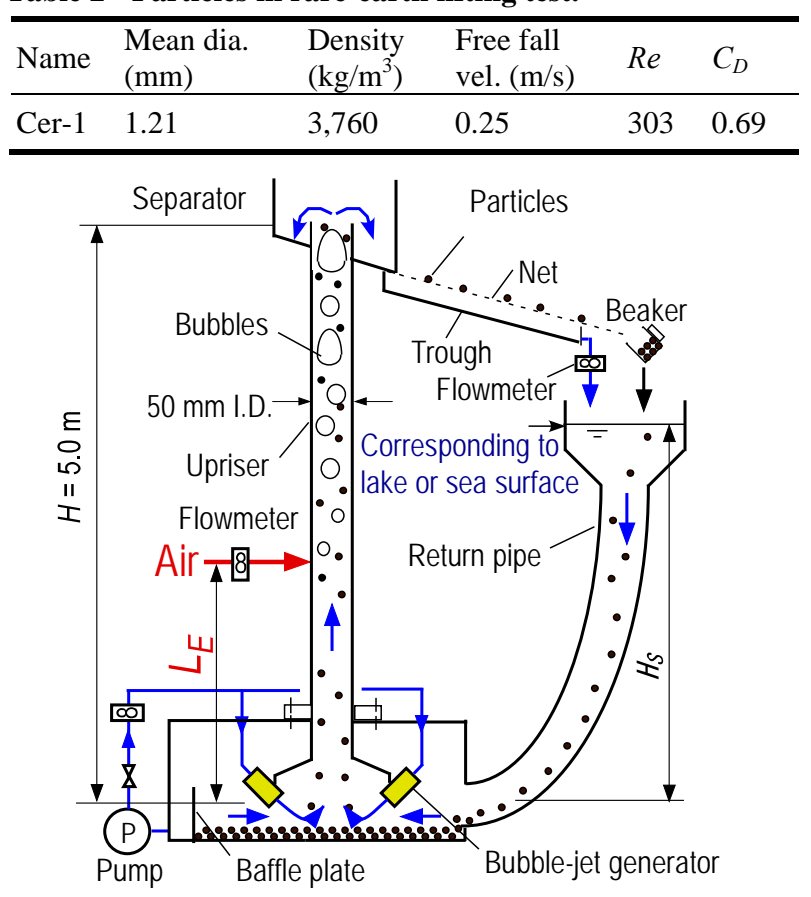

Fig. 10 Experimental apparatus (midway type). 
above the air supply position. The submergence ratio, $\sigma$, was changed as 0.76 and 0.84 , but the lifting of Cer-1 particles especially at $L_{E}=1.4 \mathrm{~m}$ at $\sigma=0.76$ was unstable, and the accuracy of the measurement seemed low. So, the data at $\sigma=0.84$ alone are described in the next chapter.

\subsection{Experimental Results}

Fig. 11 shows the present experimental data on the discharge rates of (a) the Cer-1.0 particles and (b) water when the BJT air-lift pump was operated at $\sigma\left(=H_{S} / H\right)$ $=0.84$. In order to study the effects of air supply position, the data for the bottom air supply type and two different midway air supply types are simultaneously plotted. Since the bottom type corresponds to $L_{E}=0$ in the midway type, it is cleared that the particles and water discharge rates decrease with increasing of $L_{E}$. In the midway type, the driving force of the pump is given by the density difference between the gas-liquid-solid flow part in the upriser and the water outside the upriser. Thus, the shortening of the gas-liquid-solid flow part by the elongation of $L_{E}$ lowers the pump performance. In addition, the discharge rates for each types increase with $Q_{G I}$, because the three-phase mixture density decreases with increasing of $Q_{G I}$. However, if $Q_{G I}$ is further increased, the discharge rates decrease due to the increase in wall friction inside the upriser. Thus, the data were obtained in the $Q_{G I}$ range of $Q_{G I}<5 \mathrm{l} / \mathrm{s}$.

Fig. 12 shows particle flow rate fraction data, $\beta_{S}$ (= $\left.Q_{S O} /\left(Q_{L O}+Q_{S O}\right)\right)$, against the air supply rate. In our previous study [15] for the bottom air supply type, different BJ generator with a sphere instead of an orifice was used, and the following correlation was proposed based on the experimental data for three kinds of spherical particles with different size and density together with river sand:

$$
\beta_{S}=K \cdot\left(\frac{d_{S}}{D}\right)^{-0.16}\left(\frac{\rho_{S}}{\rho_{L}}\right)^{-3.4}\left(\frac{\rho_{L} u_{S \infty x} d_{S}}{\mu_{L}}\right)^{-1.0}
$$

Here, $d_{s}$ and $D$ are the particle and the upriser diameters, $\rho$ the density, $\mu$ the viscosity, and $u_{s \infty}$ the

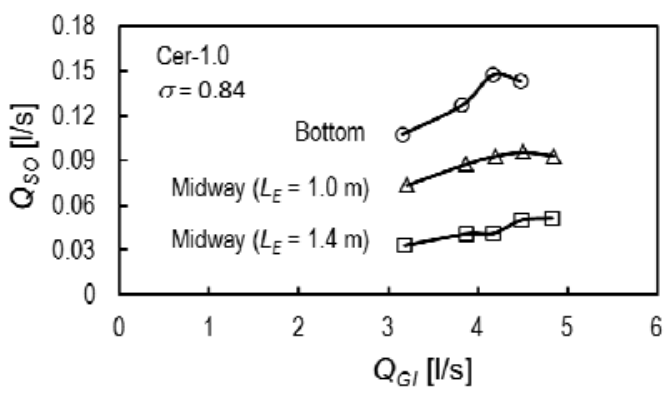

Particle

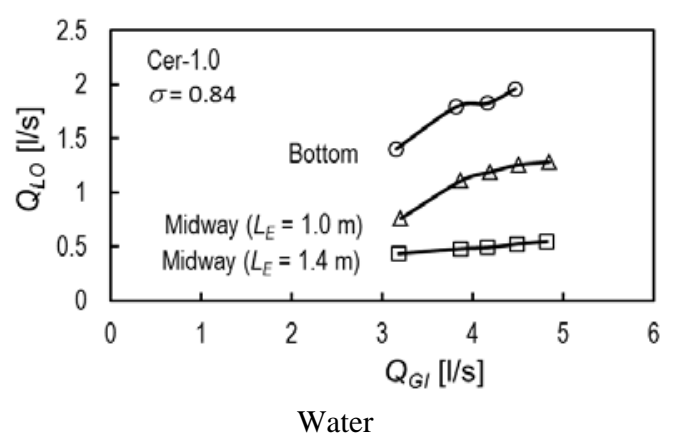

Fig. 11 Effects of air supply position on discharge rates of particles and water.

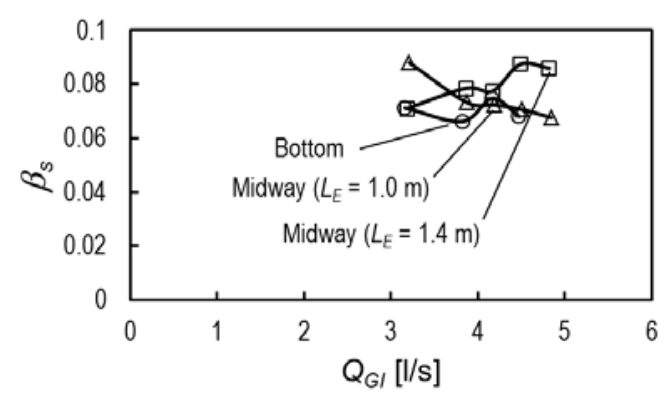

Fig. 12 Effects of air supply position on particles flow rate fraction in water-particles mixture flow.

particle falling velocity in a stagnant water pool without wall effects. The constant, $K$, for the spherical ones was 407 . The mean value of $\beta_{S}$ data in Fig. 12 is 0.077 , while the $\beta_{S}$ calculated by Eq. (3) with $K=407$ is 0.027 , thus the present $\beta_{S}$ data are about three times higher than those calculated. Since $\beta_{S}$ in Fig. 12 is almost independent of the air supply position and the air supply rate, the increase in $\beta_{S}$ in the present study must be caused by the change in the BJ generator. Thus, the present $\mathrm{BJ}$ generator is superior to the previous one for particle floatation.

Fig. 13 shows $Q_{S O}$ data against the total power needed for lifting particles, calculated as the sum of $L_{L}$ 


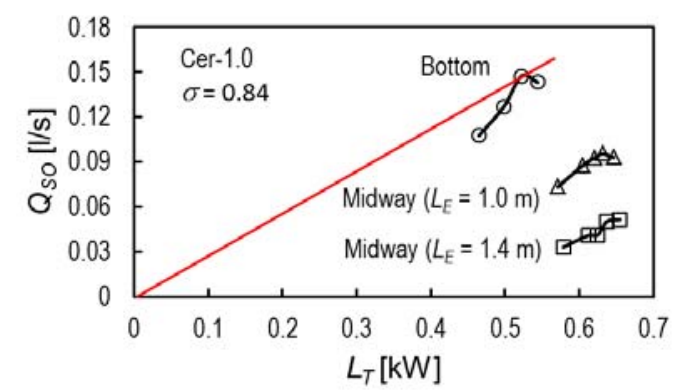

Fig. 13 Effects of air supply position on particles lifting efficiency.

and $L_{G}$ in Eqs. (1) and (2). Fig. 13 also shows a tangential line through the origin to the maximum value of the data. Since the slope of the line is steeper the better for saving energy in the operation, it is clear that the bottom air supply type is the most efficient, and the slope for the midway type of $L_{E}=1.0 \mathrm{~m}$ is about half of that for the bottom type.

\section{Numerical Simulation on Rare-Earth Lifting}

For the bottom air supply type, Sadatomi et al. [15] proposed a performance prediction model. In the model, correlations for the air-water-particles flow part alone in Yoshinaga et al.'s model [4] were used because water-particles flow part does not exist in the bottom air supply type. In addition, in order to make Yoshinaga et al.'s model complete, Eq. (3) was incorporated into the model. The basic equation of the Sadatomi et al.'s model [15] is the following momentum conservation equation:

$$
\begin{gathered}
\Delta\left(\rho_{G} j_{G} u_{G}+\rho_{L} j_{L} u_{L}+\rho_{S} j_{S} u_{S}\right)_{E-O}-d P_{E}-\frac{4}{D} \int_{E}^{O} \tau_{3} d z \\
-\int_{E}^{O}\left(\rho_{G} \varepsilon_{G}+\rho_{L} \varepsilon_{L}+\rho_{S} \varepsilon_{S}\right) g d z+\rho_{L} g H_{S}=0
\end{gathered}
$$

Here, $j$ is the superficial velocity of each phase, $\varepsilon$ the volume fraction of each phase, $\tau_{3}$ the wall shear stress for the three-phase flow, $z$ the axial distance and $g$ the gravitational acceleration. The first term in Eq. (4) is the change in momentum from the entrance to the outlet of the upriser, the second the entrance pressure loss, the third and the fourth the wall friction loss and the hydrostatic pressure loss, and the fifth the hydrostatic pressure at the upriser inlet exerted from the outside. These terms are calculated by the same equations given by Yoshinaga et al. [4] except for the second term. For the second term, by accounting for the effects of the air flow we used:

$$
d P_{E}=\zeta\left(\rho_{G} \varepsilon_{G}+\rho_{L} \varepsilon_{L}+\rho_{S} \varepsilon_{S}\right)\left(j_{G}+j_{L}+j_{S}\right)^{2} / 2
$$

Here, the inlet pressure loss coefficient, $\zeta$, was recommended as $\zeta=1.5$ [15].

For the midway air supply type, since water-particles two-phase flow exists below the air supply position, Eq. (4) must be modified as follows:

$$
\begin{array}{r}
\left(\rho_{L} j_{L} u_{L}+\rho_{S} j_{S} u_{S}\right)_{E}-\left(\rho_{G} j_{G} u_{G}+\rho_{L} j_{L} u_{L}+\rho_{S} j_{S} u_{S}\right)_{O} \\
-d P_{E}-\frac{4}{D} \int_{E}^{I} \tau_{L S} d z-\frac{4}{D} \int_{I}^{O} \tau_{3} d z-\int_{E}^{I}\left(\rho_{L} \varepsilon_{L}+\rho_{S} \varepsilon_{S}\right) g d z \\
-\int_{I}^{O}\left(\rho_{G} \varepsilon_{G}+\rho_{L} \varepsilon_{L}+\rho_{S} \varepsilon_{S}\right) g d z-d P_{I}+\rho_{L} g H_{S}=0
\end{array}
$$

Here, the first term is the liquid and the solid momentum influx to the upriser entrance, the second the gas, the liquid and the solid momentum efflux from the upriser outlet. The third term is the entrance pressure loss, the fourth and the fifth the wall shear stress respectively for the liquid-solid flow and the three-phase flow. The sixth and the seventh terms are the hydrostatic pressure loss respectively for the liquid-solid flow and the three-phase flow. The eighth term is the pressure loss due to the air supply and the ninth the hydrostatic pressure at the upriser inlet exerted from the outside. In the performance prediction, these terms are calculated by the same equations given by Yoshinaga et al. [4], and the particle flow rate fraction was taken as $\beta_{S}=0.077$, being the mean value of the present data, irrespective of the difference of the air supply type, i.e., the bottom and the midway types.

Fig. 14 compares the calculations by the above models with the present data on the discharge rates of (a) the particles and (b) water when the BJT air-lift pump was operated at $\sigma=0.84$. The broken curves are the calculations for the respective air supply types. The agreement between the calculations and the experiments is not perfect, but the trend of the data is 
well predicted by the model, i.e., $Q_{S O}$ increases with increasing of $Q_{G I}$ and with decreasing of $L_{E}$.

Since the rare-earth rich mud exists on the seabed deeper than $4,000 \mathrm{~m}$, so another simulation was conducted under the following conditions: $H=6,000 \mathrm{~m}$ for the upriser length and $H_{S}=5,940 \mathrm{~m}$ for the submerged depth, (corresponding to the submergence ratio of $\sigma=0.99$ ), $D=150$ to $300 \mathrm{~mm}$ for the riser diameter, $L_{E}=4,000 \mathrm{~m}$ to $5,000 \mathrm{~m}$ for the midway air supply position. Such $H$ and $L_{E}$ are determined by referring $H=5,300 \mathrm{~m}$ and $L_{E}=3,500 \mathrm{~m}$ in the simulation by Hatakeyama et al. [17].

Fig. 15 shows calculated results on the discharge rates of (a) Cer-10 particles and (b) water in order to know the effects of the upriser diameter. The discharge rates for the respective diameters increase with the air supply rate, $Q_{G I}$, as expected. A tangential line through the origin is drawn to the curve at $D=300 \mathrm{~mm}$. It is clear that the use of $D=300 \mathrm{~mm}$ upriser is the most efficient because the curves for other diameters are lower than the tangential line. The reason of this is that
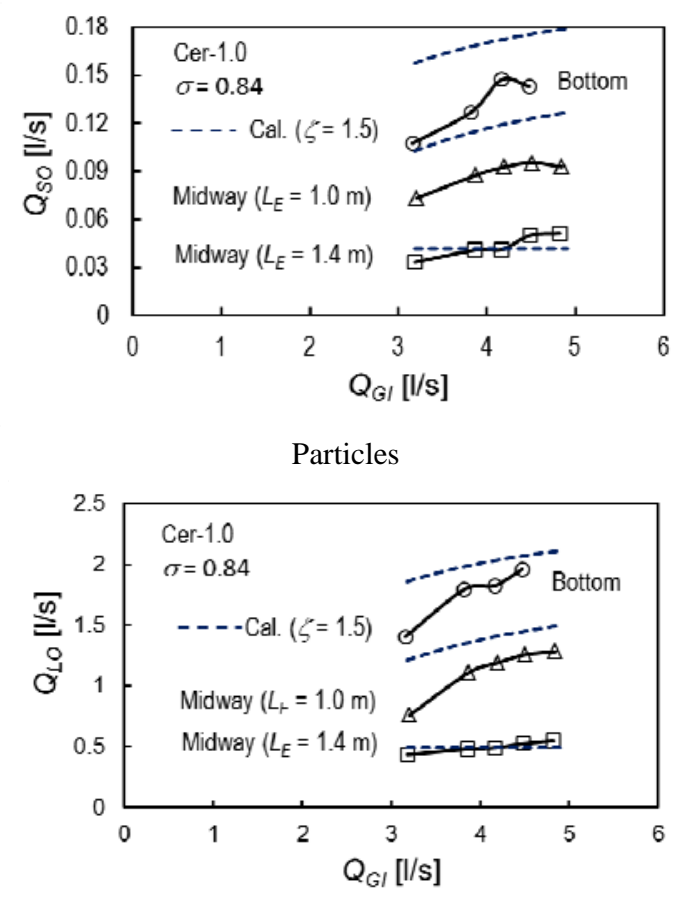

Water

Fig. 14 Comparison of discharge rates of particles and water between experiment and calculation.

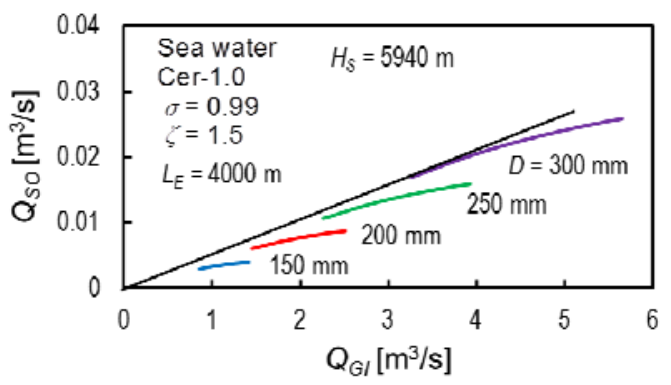

Particles

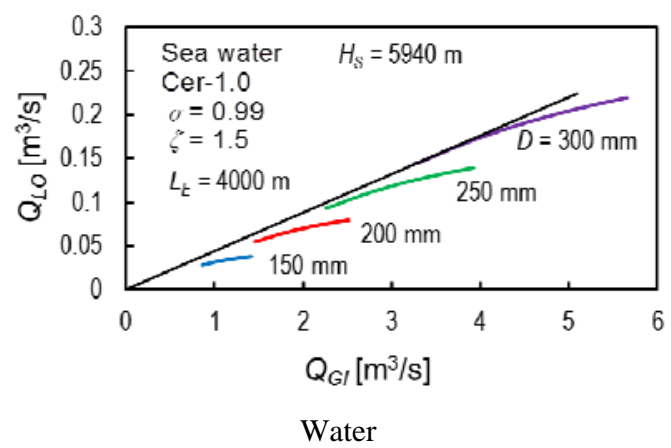

Fig. 15 Effects of upriser diameter on discharge rates of particles and water when $H=6,000 \mathrm{~m}$ and $L_{E}=4,000 \mathrm{~m}$.

the wall friction terms in Eq. (6) decreases with increasing of the upriser diameter. In addition, $Q_{G I}$ is smaller the better because the respective calculated curves approach the tangential line.

Fig. 16 shows calculated results on the discharge rates of (a) Cer-10 particles and (b) water in order to know the effects of air supply position, $L_{E}$. The curve for $L_{E}=5,000 \mathrm{~m}$ is lower than those for $4,000 \mathrm{~m}$ and $4,500 \mathrm{~m}$. Such a trend is similar to the present data in Fig. 11. $L_{E}$ is shorter the better because the gas-liquid-solid flow part length $\left(=H-L_{E}\right)$, giving driving force of the pump operation, becomes long. However, $Q_{S O}$ and $Q_{L O}$ data for $L_{E}=4,000 \mathrm{~m}$ and 4,500 $\mathrm{m}$ are nearly the same. This suggests that too short $L_{E}$ is no effective, because the wall friction pressure drop is much higher in the gas-liquid-solid flow than the liquid-solid flow. In addition, if $L_{E}$ is short, the pneumatic power supplied by a compressor, $L_{G}$, which is calculated by Eq. (2) becomes huge because $P_{G I}$ must be higher than $\rho g\left(H_{S}-L_{E}\right)$.

In order to find better air supply position, further studies are needed. One of them is to find a proper 


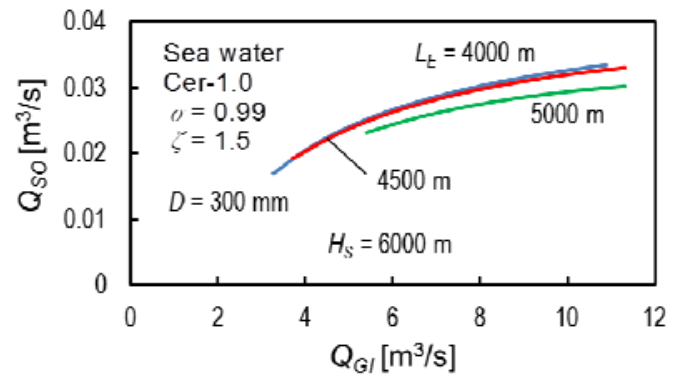

Particles

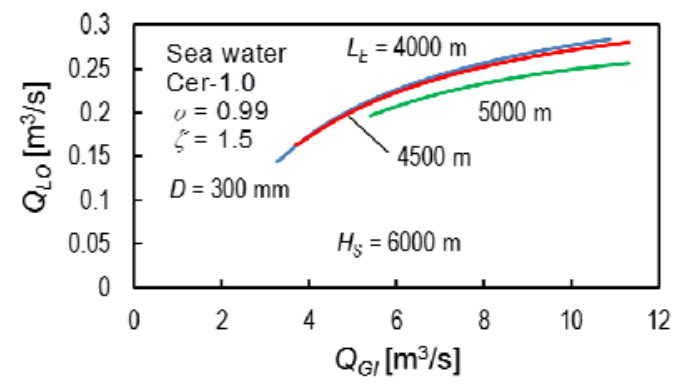

Water

Fig. 16 Effects of air supply position on discharge rates of particles and water when $H=6,000 \mathrm{~m}$ and $D=300 \mathrm{~mm}$.

upriser proportion, including air supply position which gives the highest $Q_{S O} / L_{G}$ ratio.

\section{Conclusions}

The applicability of the BJT pump for lifting seabed materials in deep sea was studied experimentally and analytically. Targets are (a) methane-hydrate rich muds on the seabed about $200 \mathrm{~m}$ in depth around Japan islands and (b) rare-earth rich muds on the seabed deeper than 4,000 m around Minami-Torishima islands in the Pacific Ocean. The main findings are as follows:

(1) In the methane-hydrate lifting simulation experiment, the discharge rate became 2.8 times higher for VC-3.9 (simulated methane-hydrate) than the sand by selecting optimum water and air supply rates;

(2) In the rare-earth lifting simulation experiment, the midway air supply was tested in addition to the bottom air supply. The discharge rates of Cer-1.0 particles, simulating rare-earth mud, and water decreased as the distance from the air supply position to the upriser bottom increased;

(3) The trend of the data on the rare-earth lifting simulation experiment was well predicted by our previous performance prediction model and the present modified one for the midway air supply type;

(4) The model mentioned above was used to simulate the discharge rates of Cer-1.0 and water from a seabed in 6,000 $\mathrm{m}$ deep in order to study the effects of the upriser diameter and the distance from air supply position to the upriser bottom. Their effects on the pump performance were clarified.

\section{Acknowledgments}

The authors appreciate very much for a financial support from JSPS (JSPS KAKENHI Grant Number 26420117).

\section{References}

[1] Kawashima, T., Noda, K., Masuyama, T., and Oda, S. 1975. "Hydraulic Transport of Solids by Air Lift Pump." Journal of MMIJ 91: 765-72. (in Japanese)

[2] Weber, M., and Dedegil, M. Y. 1976. "Transport of Solids According to the Air-Lift Principle.” In Proc. 4th Int. Conf. on the Hydraulic Transport of Solids in Pipes, H1-1-23-X93-94.

[3] Kato, H., Miyagawa, T., Tamiya, S., and Iwasaki, T. 1975. “A Study of an Air-Lift Pump for Solid Particles.” Bull. of JSME 18: 286-94.

[4] Yoshinaga, T., and Sato, Y. 1996. "Performance of an Air-Lift Pump for Conveying Coarse Particles.” Int. J. Multiphase Flow 22: 223-38.

[5] Fujimoto, H., Ogawa, S., Takuda, H., and Hatta, N. 2003. "Operation Performance of a Small Air-Lift Pump for Conveying Solid Particles.” Trans. ASME, J. Energy Res. Technol. 125: 17-25.

[6] Fujimoto, H., Murakami, S., Omura, A., and Takuda, H. 2004. "Effect of Local Pipe Bends on Pump Performance of a Small Air-Lift System in Transporting Solid Particles.” Int. J. Heat and Fluid Flow 25: 996-1005.

[7] Fujimoto, H., Nagatani, T., and Takuda, H. 2005. "Performance Characteristics of a Gas-Liquid-Solid Air-Lift Pump.” Int. J. Multiphase Flow 31: 1116-33.

[8] Samaras, V. C., and Margaris, D. P. 2009. "Predicting Three-Phase Air-Lift Pump Performance.” Int. Review of Mechanical Engineering 3: 339-44.

[9] Samaras, V. C., and Margaris, D. P. 2010. "Investigating Experimentally Flow Regimes in Three-Phase Air-Lift Pumps.” Int. Review of Mechanical Engineering 4: 726-32.

[10] Sadatomi, M. 2012. Japanese Patent No. 5007468. 
[11] Sadatomi, M., Kawahara, A., Matsuyama, F., and Kimura, T. 2007. "An Advanced Micro-bubble Generator and Its Application to a Newly Developed Bubble-Jet-Type Air-Lift Pump.” Multiphase Science and Technology 19: 323-42.

[12] Sadatomi, M., Kawahara, A., Kimura, T., and Nakao, J. 2007. "Development of a Bubble-Jet-Type Air-Lift-Pump-Its Feasibility Test.” In Proc. of 6th Int. Conf. on Multiphase Flow (ICMF 2007), Paper \# S7_Mon_B_1.

[13] Sadatomi, M., Kawahara, A., Kimura, T., and Nakao, J. 2008. "Development of a Bubble-Jet-Type Air-Lift-Pump-Feasibility Test and Performance Prediction.” Transactions of JSME Part B 74: 1263-9. (in Japanese)

[14] Sadatomi, M., Kawahara, A., and Goto, T. 2010. "Experiment and Performance Prediction of a
Bubble-Jet-Type Air Lift Pump for Dredging Sediments.” In Proc. of 7th Int. Conf. on Heat Transfer, Fluid Mechanics and Thermo-dynamics, 6 pages in CD-ROM.

[15] Sadatomi, M., Kawahara, A., and Nishiyama, T. 2012. "Experiment and Performance Prediction of Bubble-Jet Type Air-Lift Pump for Dredging Sediments on Sea and Lake Beads." Advances in Fluid Mechanics and Heat \& Mass Transfer, ISBN 978-1-61804, 311-6.

[16] Kato, Y., Fujinaga, K., Nakamura, K., Takaya, Y., Kitamura, K., Ohta, J., Toda, R., Nakashima, T., and Iwamori, H. 2011. "Deep-Sea Mud in the Pacific Ocean as a Potential Resource for Rare-Earth Elements.” Nature Geoscience 4: 535-9.

[17] Hatakeyama, N., Takahashi, H., Saito, T., and Masuyama, T. 1999. "A Numerical Simulation of Unsteady Two-Phase Gas-Liquid Flow in a Large Scale Air-Lift Pump.” Sigen-to-Sozai 115: 437-42. (in Japanese) 